\title{
Non-aneurysmal and non-traumatic subarachnoid hemorrhage after attempted suicide by incomplete hanging
}

elSSN: $2383-4625$

\section{Tae Hu Kim, Soo Hoon Lee, Dong Hoon Kim, Seong Chun Kim, Wonyoungyong Jin, So Yeon Kim, Sang Heon Shin, Sang Bong Lee}

Department of Emergency Medicine, Gyeongsang National University Hospital, Gyeongsang National University School of Medicine, Jinju, Korea

Hanging is a common method of suicide that is being reported more frequently in many countries. Several complications including injuries to the cervical spine, neck vessels, and brain can occur after attempted suicide by hanging. There are only a few reports of brain computed tomography and magnetic resonance imaging of hanging victims. The most common abnormality was diffuse cerebral edema. A subarachnoid hemorrhage is an atypical complication by suicidal hanging. We report a case of a female patient who presented to an emergency department with altered mental status after attempting suicide by incomplete hanging. The patient was diagnosed with a non-aneurysmal and non-traumatic subarachnoid hemorrhage. This case shows that spontaneous subarachnoid hemorrhage can develop due to a sudden elevation of intracranial pressure, as occurs with hanging.

Keywords Hanging; Strangulation; Subarachnoid hemorrhage; Suicide

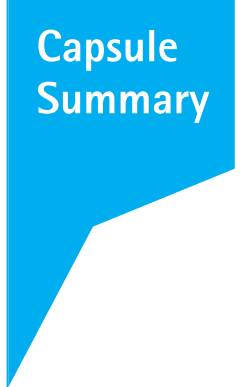

What is already known

Several complications including injures to the cervical spine, neck vessels, and brain can occur after attempted suicide by hanging.

What is new in the current study

Spontaneous subarachnoid hemorrhage can develop due to a sudden elevation of intracranial pressure, as occurs with hanging.
Received: 26 November 2016 Revised: 30 November 2016 Accepted: 28 December 2016

Correspondence to: Soo Hoon Lee Department of Emergency Medicine, Gyeongsang National University Hospital, Gyeongsang National University School of Medicine, 79 Gangnam-ro, Jinju 52727, Korea E-mail:ssoon0702@naver.com

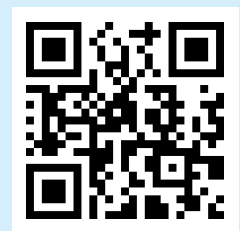

How to cite this article:

Kim TH, Lee SH, Kim DH, Kim SC, Jin W, Kim SY, Shin SH, Lee SB. Non-aneurysmal and non-traumatic subarachnoid hemorrhage after attempted suicide by incomplete hanging. Clin Exp Emerg Med 2017;4(1):56-59.

This is an Open Access article distributed under the terms of the Creative Commons Attribution Non-Commercial License (http:// creativecommons.org/licenses/by-nc/3.0/). 


\section{INTRODUCTION}

Hanging is a common method of suicide that is being reported more frequently in many countries. In addition, it has a higher success rate compared to other common methods of suicide, such as self-poisoning and self-mutilation. ${ }^{1,2}$ Hanging occurs when pressure is exerted on the neck and then increased by the weight of the victim's body. Hangings are referred to as complete or incomplete depending on whether or not the feet are in contact with the ground. Although the mechanism of death may differ depending on the method of hanging, death from non-judicial hanging probably results from airway obstruction (suffocation) or vascular occlusion. ${ }^{3}$

Several complications including injuries to the cervical spine, neck vessels, and brain can occur by hanging. ${ }^{4-6}$ Many patients are admitted to the emergency department (ED) after attempting suicide by hanging, and their symptoms range from mild with an alert state of consciousness to severe with decreased mental status or even cardiac arrest. Brain imaging such as brain computed tomography (CT) or magnetic resonance imaging (MRI) is recommended if the patient's neurologic symptoms change. The main reported cause of brain damage in hanging is hypoxia, which causes cerebral infarction, venous hypertension, and dural sinus thrombosis. ${ }^{7.8}$

We report a case of a female patient who presented to an ED with altered mental status after attempting suicide by incom- plete hanging. The patient was diagnosed with a non-aneurysmal and non-traumatic subarachnoid hemorrhage (SAH) that occurred after she attempted suicide by incomplete hanging.

\section{CASE REPORT}

A 33-year-old woman was admitted to the ED with altered mental status after suicidal hanging. She hung herself from a towel rack with a bath towel. She hung for about 10 minutes and her feet were in contact with the ground, according to the statement by her husband. On admission, her vitals were a blood pressure of 140/90 $\mathrm{mmHg}$, heart rate of 134 beats/min, respiratory rate of 26 breaths $/ \mathrm{min}$, and body temperature of $36^{\circ} \mathrm{C}$. The patient had no past medical history. Physical examination revealed she was in a semicomatose state, with a score of 8 on the Glasgow Coma Scale. Her pupils were normal and equal in size and reacted to light promptly. No further abnormalities were revealed in other neurological examinations. The only outer, visible signs of trauma were the ligature hanging marks around her neck. The patient was intubated for risk of airway obstruction.

Brain CT was performed and showed a SAH in the quadrigeminal plate cistern of the brain (Fig. 1A). The cervical spine CT showed no abnormalities. On day 2, the patient became alert and was extubated. A neurologic examination was performed to evaluate mental status and to identify any motor weaknesses, sensory
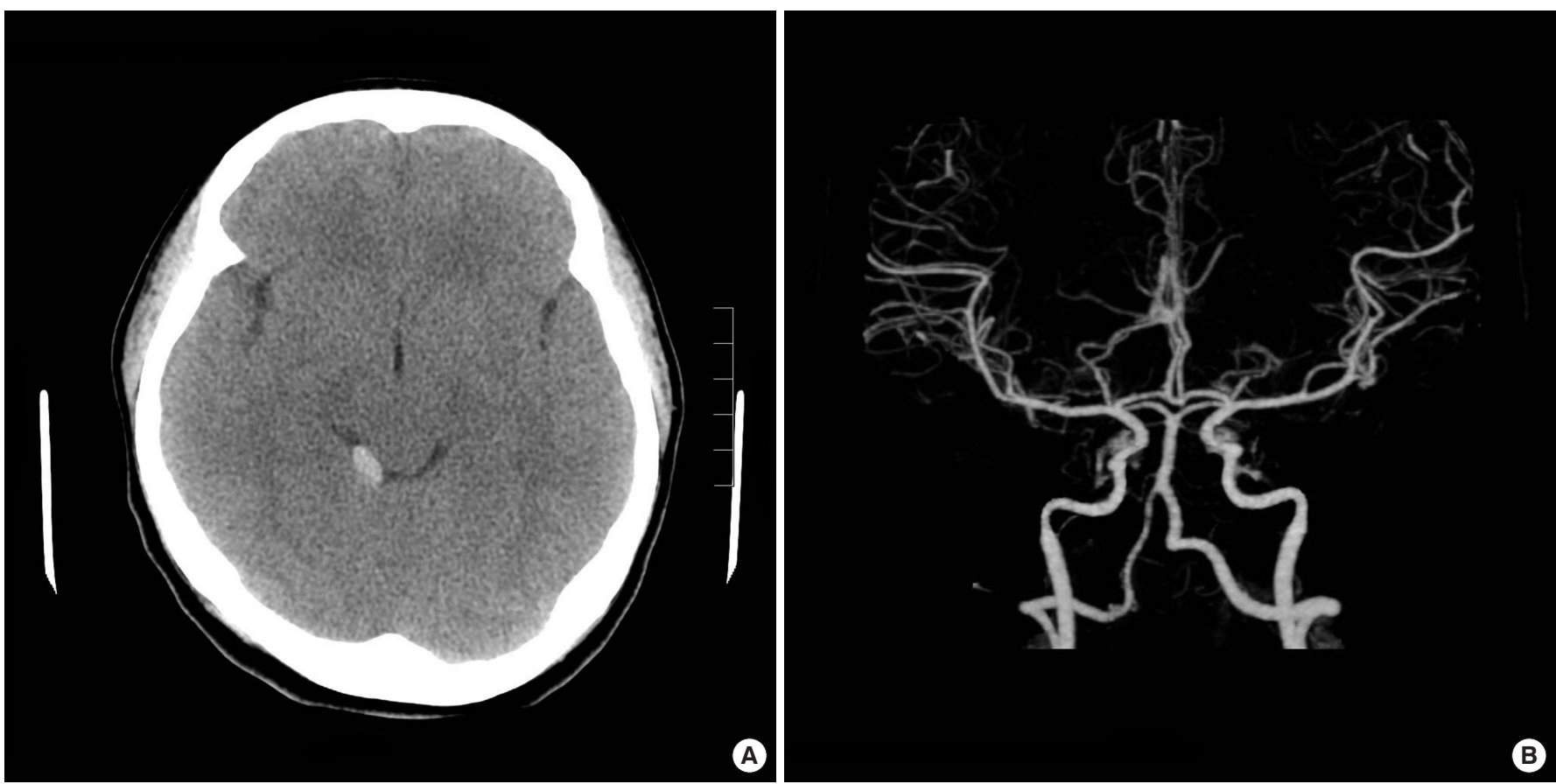

Fig. 1. (A) A brain computed tomography scan of a patient demonstrating acute subarachnoid hemorrhage in quadrigeminal plate cistern. (B) A cerebral computed tomography angiography scan of a patient demonstrating no vascular abnormality such as cerebral aneurysm or arteriovenous malformation. 
disturbances, or other neurologic abnormalities. The patient was alert, and no other abnormalities were observed upon neurological examination. On day 4, we performed another brain CT. The SAH did not change in comparison with the previous $\mathrm{CT}$, and there was no evidence of hypoxic brain injury. On day 5, we performed a brain angiography $\mathrm{CT}$ and transfemoral cerebral angiography to identify any abnormal vascular features, such as a cerebral aneurysm or arteriovenous malformation. No vascular abnormalities were found (Fig. 1B). The patient was treated conservatively for 6 days and then discharged.

\section{DISCUSSION}

This case report demonstrates that non-aneurysmal and non-traumatic SAH can occur after suicidal hanging. Most SAHs are caused by trauma or a ruptured aneurysm; however, in approximately $15 \%$ to $20 \%$ of cases of spontaneous SAH no aneurysm is detected on the first brain angiography. ${ }^{9}$ In such cases, SAHs can be related to a variety of conditions including arteriovenous malformations, cerebral artery dissection, coagulopathies, moyamoya syndrome, mycotic aneurysm, neoplasm, pituitary apoplexy, vasculopathy, brain tumors, and the use of sympathomimetic drugs (particularly amphetamine, cocaine, phenylpropanolamine, and pseudoephedrine). The SAH limited to the perimesencephalic area found in this patient could have been attributed to one of the aforementioned conditions, as it is not a common location for a SAH due to head trauma or cerebral aneurysm.

There are only a few reports of brain $\mathrm{CT}$ and MRI of hanging victims. $^{7,8,10,11}$ The most common abnormality they described was diffuse cerebral edema. The other brain CT and MRI abnormalities included round bilateral low density areas in the globi pallidi; diffuse swelling; and symmetrical low density areas in the basal ganglia, thalamus, and midbrain; subcortical hematomas with a $\mathrm{SAH}$; and multiple infarctions due to dissection of the carotid artery. Interestingly, intracranial hemorrhages including subcortical and subdural, and SAH could be seen. A SAH is an atypical complication that occurs after hanging; although, theoretically, this may reflect spontaneous rupture of vessels within a high-pressure venous system created by vascular obstruction. In complete hangings, the rapid and forceful appliance of gravitational pressure on the neck constricts both the arteries and the veins, typically with no subsequent visible petechial hemorrhages. However, in incomplete hangings, less gravitational pressure is exerted on the neck resulting in no arterial and only partial venous compression; and hence, facial congestion, cyanosis, and petechial hemorrhages. ${ }^{12}$ Most previous cases associated with SAHs occurred after judicial or homicidal hanging, or a head injury related to trauma. In the current case, the patient did not have a history of head trauma, and the transfemoral cerebral angiography did not show any vascular abnormalities such as cerebral aneurysm or arteriovenous malformation.

In summary, many patients are admitted to the ED after attempting suicide by hanging, and their symptoms range from mild with an alert state of consciousness to severe with a decreased mental state or even cardiac arrest. Brain imaging, such as a brain CT or MRI, is recommended if the patient's neurologic symptoms change. This case shows that spontaneous SAH can develop due to a sudden elevation of intracranial pressure, as occurs with hanging.

\section{CONFLICT OF INTEREST}

No potential conflict of interest relevant to this article was reported.

\section{REFERENCES}

1. Farmer R, Rohde J. Effect of availability and acceptability of lethal instruments on suicide mortality. An analysis of some international data. Acta Psychiatr Scand 1980;62:436-46.

2. Meel BL. A study on the incidence of suicide by hanging in the sub-region of Transkei, South Africa. J Clin Forensic Med 2003; 10:153-7.

3. Iserson KV. Strangulation: a review of ligature, manual, and postural neck compression injuries. Ann Emerg Med 1984;13: 179-85.

4. Martin MJ, Weng J, Demetriades D, Salim A. Patterns of injury and functional outcome after hanging: analysis of the $\mathrm{Na}-$ tional Trauma Data Bank. Am J Surg 2005;190:836-40.

5. Nichols SD, McCarthy MC, Ekeh AP, Woods RJ, Walusimbi MS, Saxe JM. Outcome of cervical near-hanging injuries. J Trauma 2009;66:174-8.

6. Salim A, Martin M, Sangthong B, Brown C, Rhee P, Demetriades D. Near-hanging injuries: a 10-year experience. Injury 2006;37: 435-9.

7. Brancatelli G, Sparacia G, Midiri M, D'Antonio V, Sarno C, Lagalla R. Brain damage in hanging: a new CT finding. Neuroradiology 2000;42:209-10.

8. Nakajo M, Onohara S, Shinmura K, et al. Computed tomography and magnetic resonance imaging findings of brain damage by hanging. J Comput Assist Tomogr 2003;27:896-900.

9. Rinkel GJ, van Gijn J, Wijdicks EF. Subarachnoid hemorrhage without detectable aneurysm. A review of the causes. Stroke 1993;24:1403-9. 
10. Lee BK, Jeung KW, Lee HY, Lim JH. Outcomes of therapeutic hypothermia in unconscious patients after near-hanging. Emerg Med J 2012;29:748-52.

11. Solhi H, Pazoki S, Mehrpour O, Alfred S. Epidemiology and prognostic factors in cases of near hanging presenting to a re- ferral hospital in Arak, Iran. J Emerg Med 2012;43:599-604.

12. Rasmussen ER, Larsen PL, Andersen $K$, Larsen M, Qvortrup $K$, Hougen HP. Petechial hemorrhages of the tympanic membrane in attempted suicide by hanging: a case report. J Forensic Leg Med 2013;20:119-21. 